\title{
Article
}

\section{Associations between serum relaxin 2, aneurysm formation/size and severity of atherosclerosis: a preliminary prospective analysis}

\author{
Konstantinos PAPOUTSIS ${ }^{1, \#}$, Alkistis KAPELOUZOU ${ }^{2, \#}$, Diamantis I TSILIMIGRAS ${ }^{1, \#}$, Nikolaos PATELIS ${ }^{1, *}$, Georgios KOU- \\ VELOS $^{3}$, Dimitrios SCHIZAS ${ }^{1}$, Ioannis KARAVOKYROS ${ }^{1}$, Sotirios GEORGOPOULOS ${ }^{1}$ \\ ${ }^{1}$ First Department of Surgery, Vascular Unit, Laiko General Hospital, National \& Kapodistrian University of Athens, 11527 Greece; \\ ${ }^{2}$ Clinical, Experimental Surgery \& Translational Research, Biomedical Research Foundation Academy of Athens, 11527 Greece; \\ ${ }^{3}$ Department of Vascular Surgery, University Hospital of Larissa, 41500 Greece
}

\begin{abstract}
Serum relaxin $2(R L 2)$ is a pleiotropic hormone that acts on various organs and systems, particularly the cardiovascular system. Although RL2 seems to upregulate the synthesis of nitric monoxide (NO) and matrix metalloproteinase (MMP)-2 and -9, current literature on its role in atherosclerosis and aneurysm formation is scarce. The aim of this study was to investigate the levels of serum RL2 in patients with an arterial aneurysm as well as in atherosclerotic patients, and correlate them with the severity of their related vascular disease. A total of 53 subjects were enrolled in this study: 37 patients were scheduled to undergo surgery: 21 patients for different forms of atherosclerotic disease (ATH), 16 patients for an arterial aneurysm (AA), 6 patients for undergoing temporal artery biopsy (TAB), and 10 healthy blood donors (HBD) served as the control groups. RL2 was measured using enzymelinked immunosorbent assay. RL2 was significantly higher in AA patients compared to ATH $(P<0.01)$, TAB $(P<0.001)$ and HBD $(P<0.01)$. No significant difference was found between the ATH and TAB groups $(P>0.05)$. In addition, ATH and AA patients were further subdivided based on the severity of their disease. Serum RL2 was progressively increased in patients with arterial aneurysms, showing a positive relationship with the size of the aneurysmatic dilatation. By contrast, the RL2 level was inversely related to the severity of the atherosclerotic disease. Studies with a larger cohort incorporating a consistent study population are warranted to verify our results and shed light on the mechanistic background of these processes.
\end{abstract}

Keywords: relaxin; aneurysm; atherosclerosis; aortic dilatation; metalloproteinase; MMP

Acta Pharmacologica Sinica (2018) 39: 1243-1248; doi: 10.1038/aps.2018.8; published online 22 Mar 2018

\section{Introduction}

Relaxin 2 (RL2) is a peptide hormone that acts on various organs and systems, particularly the cardiovascular system ${ }^{[1]}$. RL2 is structurally related to insulin, and like insulin, its mature peptide derives from a pro-hormone after processing by prohormone convertases ${ }^{[2-4]}$. RL2 binds to and activates relaxin family peptide receptor-1 (RFXP-1), a G proteincoupled receptor (GPCR) that triggers signaling pathways through the generation of second messengers such as nitric oxide (NO) and cyclic adenosine monophosphate (cAMP) and the stimulation of the phosphorylation of mitogen-activated protein $(\mathrm{MAP})$ kinases $^{[3-6]}$. Interestingly, reduced NO synthe-

\footnotetext{
\# These authors contributed equally to this work.

*To whom correspondence should be addressed.

E-mail patelisn@gmail.com

Received 2017-10-28 Accepted 2018-01-07
}

sis and bioavailability due to direct quenching by superoxide anion can contribute to cardiovascular diseases such as atherosclerosis $^{[7]}$.

Apart from reproductive tissues, RL2 has been detected in human brain and heart ${ }^{[3,4]}$, and RFXP-1 is expressed in rodents in the aorta, vena cava, mesenteric artery, mesenteric vein, femoral artery, femoral vein, small pulmonary arteries and small renal arteries ${ }^{[3,8,9]}$. Meanwhile, RL2 upregulates the synthesis of matrix metalloproteinase (MMP)-2 and -9 in various tissues, including arteries ${ }^{[10-12]}$. The role of MMPs in aneurysm formation is well established ${ }^{[13-17]}$. MMPs induce the degradation of the vessel wall extracellular matrix. In that context, RL2 may theoretically be implicated in various vascular diseases; yet, current literature on its role in atherosclerosis and aneurysm formation is scarce ${ }^{[18-22]}$.

The aim of the present study was to investigate the level of serum RL2 in patients with an arterial aneurysm (AA) and 
correlate them with the size of the aneurysm and the related clinical presentation of the patient (whether symptomatic or not). We also investigated whether serum RL2 in atherosclerotic (ATH) patients was correlated with the clinical severity of the disease (permanent or not ischemic manifestations from organs that are supplied from the atherosclerotic vessels). Both AA and ATH patients were compared with control groups consisting of healthy blood donors (HBD) and patients undergoing temporal artery biopsy (TAB).

\section{Materials and methods}

\section{Study population}

A total of 53 subjects were enrolled in our study; 37 patients were scheduled to undergo surgery at Laiko Hospital in Athens, Greece, whereas 6 patients undergoing temporal artery biopsy (TAB) and 10 healthy blood donors (HBD) served as the control groups. The study was conducted in accordance to the principles outlined in the Declaration of Helsinki, and all subjects signed an informed consent for the use of their clinical and laboratory results for scientific purposes under the condition of anonymity. In brief, our interventional group consisted of 21 patients undergoing surgery for different forms of atherosclerotic disease and 16 patients undergoing surgery for an arterial aneurysm.

\section{Study groups and sub-categorization ATH group}

The ATH group included patients with extracranial carotid artery disease $(n=12)$ or peripheral arterial disease $(n=9)$. These patients were further subcategorized according to the clinical severity of their disease into three groups: 1) atherosclerosis group 1 (ATH1, $n=10)$ : patients with moderate functional consequences [asymptomatic internal carotid stenosis $>70 \%$ or claudication (Rutherford stage 3)]; 2) atherosclerosis group 2 (ATH2, n=6): patients with severe functional consequences [transient ischemic attack or rest pain (Rutherford stage 4)]; and 3) atherosclerosis group 3 (ATH3, n=5): patients with permanent disability [stroke or tissue loss (Rutherford stage 5-6)]. In patients with extracranial carotid artery disease, an experienced neurologist determined neurologic status, whereas the degree of carotid stenosis was determined by the appropriate imaging modality [digital subtraction angiography (DSA); computed tomographic angiography (CTA); or magnetic resonance angiography (MRA)] using the NASCET method ${ }^{[23]}$. Patients with peripheral arterial disease were categorized according to the revised version of recommended standards for reports dealing with lower-extremity ischemia $^{[24]}$.

\section{$A A$ group}

AA group patients had an abdominal aortic aneurysm (AAA) $(n=13)$, thoracic aortic aneurysm (TAA) $(n=1)$, internal iliac aneurysm (IIA) $(n=1)$ or popliteal aneurysm (PA) $(n=1)$ and were further categorized according to the size and clinical presentation of their aneurysm into three subgroups: 1) aneurysm group 1 (AA1) $(n=5)$ : patients with an asymptomatic aneu- rysm of a diameter $250 \%-300 \%$ of that of the normal artery; 2 ) aneurysm group 2 (AA2) ( $n=4)$ : patients with an asymptomatic aneurysm of a diameter $300 \%-350 \%$ of that of normal artery and 3) aneurysm group 3 (AA3) (n=7): patients with either a symptomatic aneurysm of any diameter or an asymptomatic aneurysm of a diameter $>350 \%$ of that of normal artery. Representative diameters of normal adult arteries as they are presented in the suggested standards for reporting on arterial aneurysms ${ }^{[25]}$ were used for AA group categorization. Aneurysm size was determined by CTA or MRA, and a patient was categorized as symptomatic based on his/her symptoms alone, regardless of the existence of rupture. Although we are not aware of any report using the above-mentioned categorization, this approach was utilized since aneurysm size is by definition proportional to the diameter of normal artery. In that context, all patients in the AA group had dilatation of various arterial segments, while the clinical severity of the disease was directly related to the presence of symptoms.

\section{$T A B$ and $H B D$ control groups}

TAB group patients $(n=6)$ were admitted for prolonged fever, and after an extensive diagnostic work-up, a temporal artery biopsy was deemed necessary. Exclusion criteria were a pathology report of temporal arteritis and a history or clinical signs of arterial aneurysm, extracranial carotid artery disease or peripheral arterial disease. Finally, the HBD group $(n=10)$ included healthy individuals participating in blood donation on a voluntary basis without history or clinical signs of an arterial aneurysm, extracranial carotid artery disease or peripheral arterial disease.

\section{Blood analysis}

Blood samples were collected by venipuncture after subjects were fasted overnight. In patients undergoing emergent operation, such as ruptured aneurysm repair and life-saving amputation, the blood samples were collected during induction of anesthesia. The samples clotted for $30 \mathrm{~min}$ at room temperature before centrifugation for $15 \mathrm{~min}$ at $1000 \times \mathrm{g}$ at $4{ }^{\circ} \mathrm{C}$ (Hettich Rotina 35R centrifuge; Hettich GmbH \& Co KG, Tuttlingen, Germany). Finally, serum was collected and stored at $-20^{\circ} \mathrm{C}$ until analysis was performed in duplicate; dilution was assessed as per protocol. Using a commercially available enzyme immunoassay kit (DRL200, R\&D systems, Minneapolis, USA), we measured serum RL2 according to the protocol of the manufacturer with an ELISA reader system (Spectramax 190; Molecular Devices, Sunnyvale, Calif, USA). Full blood count and clinical chemistry analysis were determined using a standard method (Sysmex XE-5000, Sysmex Europe GmbH, Norderstedt, Germany and Siemens Advia 1800, Siemens Healthcare $\mathrm{GmbH}$, Erlangen, Germany).

\section{Statistical analysis}

The results are presented as the mean \pm standard deviation (SD). Statistical significance for two-group comparisons was calculated using Student's unpaired-samples $t$-test. One-way ANOVA was performed for multiple comparisons between 
measured parameters. A $P$-value $<0.05$ was considered statistically significant. All statistical calculations were performed using GraphPad Prism version 4.03 (GraphPad Inc, CA, USA).

\section{Results}

\section{Baseline characteristics}

Demographics, smoking status, current medication and concurrent diseases of the study cohort are summarized in Table 1. Apart from the HBD group (mean age: $43.5 \pm 3.41$ ), all subjects underwent baseline preoperative hematologic and biochemistry tests, including full blood count, renal and liver function tests. Renal and liver tests were normal in all groups, and none of the patients presented with acute heart failure. All patients were stable without signs of septic or hypovolemic shock, even in emergent cases of gangrene or aneurysm rupture, with only two exceptions. One female patient (ATH group) undergoing emergent above-knee amputation was septic during blood sample collection. Additionally, a male participant from the AA group, with a history of kidney transplantation, had mild renal function impairment and presented with a ruptured aneurysm of his left common iliac artery. In both cases, no differences were found in serum RL2 compared to the other patients in their respective subgroups.

Overall, AA, ATH and TAB groups were matched for gender, age, medication, history as well as renal and liver function tests. The HBD group, although matched with the ATH and AA groups for gender and smoking status, represented a different population group that was approximately 25 years younger. As defined by the inclusion criteria, no one in the
HBD group had a history of concomitant disease, and no one received any medication.

\section{Serum RL2 in all study groups}

The AA group's serum RL2 (49.39 $\pm 8.620 \mathrm{pg} / \mathrm{mL})$ was significantly increased compared to the ATH group (16.22 \pm 4.697 $\mathrm{pg} / \mathrm{mL}, P<0.01)$, TAB group $(15.86 \pm 4.286 \mathrm{pg} / \mathrm{mL}, P<0.01)$ and HBD group $(10.32 \pm 1.354 \mathrm{pg} / \mathrm{mL}, P<0.01)$ (Figure 1$)$. No significant difference was observed between ATH and TAB $(P>0.05)$. Both ATH $(P<0.01)$ and TAB $(P<0.01)$ patients had significantly increased serum RL2 compared to HBD.

\section{Serum RL2 in subcategorized study groups}

Further statistical analysis in AA patients after categorization into subgroups, as shown in Figure 2, revealed that serum RL2 increased proportionally to the size and the severity of the clinical presentation of the patients. The AA1 group (38.06 \pm 2.7 $\mathrm{pg} / \mathrm{mL}$ ) had considerably lower serum RL2 compared to the AA2 group $(49.99 \pm 2.1 \mathrm{pg} / \mathrm{mL}, P<0.01)$; and AA3 group (57.1 $\pm 1.1 \mathrm{pg} / \mathrm{mL}, P<0.01)$. All AA subgroups had significantly higher serum RL2 than all ATH subgroups $(P<0.01)$, TAB patients $(P<0.001)$, and HBD group $(P<0.01)$.

Further statistical analysis in the ATH subgroup showed that serum RL2 level was inversely correlated with the clinical severity of atherosclerosis; RL2 was higher in the ATH1 $(20.65 \pm 1.419 \mathrm{pg} / \mathrm{mL})$ compared to the ATH2 group $(13.61 \pm 2.027 \mathrm{pg} / \mathrm{mL}, P<0.01)$ and the ATH3 group $(10.50 \pm 0.6044 \mathrm{pg} / \mathrm{mL}, P<0.01)$. In patients with permanent ischemic damage from target organs (ATH3 group), serum

Table 1. Subject baseline characteristics.

\begin{tabular}{|c|c|c|c|c|c|c|c|c|c|c|}
\hline & $\begin{array}{l}\text { ATH TOTAL } \\
n=21\end{array}$ & $\begin{array}{l}\text { ATH } 1 \\
n=10\end{array}$ & $\begin{array}{l}\text { ATH } 2 \\
n=6\end{array}$ & $\begin{array}{l}\text { ATH } 3 \\
n=5\end{array}$ & $\begin{array}{l}\text { AA TOTAL } \\
n=16\end{array}$ & $\begin{array}{l}\text { AA } 1 \\
n=5\end{array}$ & $\begin{array}{l}\text { AA } 2 \\
n=4\end{array}$ & $\begin{array}{l}\text { AA } 3 \\
n=7\end{array}$ & $\begin{array}{l}\text { TAB } \\
n=6\end{array}$ & $\begin{array}{l}\mathrm{HBD} \\
n=10\end{array}$ \\
\hline Gender (Male\%) & 76.2 & 70 & 83.3 & 80 & 100 & 100 & 100 & 100 & 66.6 & 70 \\
\hline Age (mean $\pm S D)$ & $\begin{array}{l}69.52 \pm \\
14.14\end{array}$ & $\begin{array}{l}70.4 \pm \\
10.1\end{array}$ & $\begin{array}{l}71 \pm \\
14.5\end{array}$ & $\begin{array}{l}62.7 \pm \\
23.9\end{array}$ & $\begin{array}{l}69.73 \pm \\
8.697\end{array}$ & $\begin{array}{l}63 \pm \\
6.9\end{array}$ & $\begin{array}{l}68.3 \pm \\
9\end{array}$ & $\begin{array}{l}75.1 \pm \\
6.6\end{array}$ & $\begin{array}{l}69.67 \pm \\
11.31\end{array}$ & $\begin{array}{l}43.50 \pm \\
3.408\end{array}$ \\
\hline $\begin{array}{l}\text { Smoker (Current } \\
\text { or former \%) }\end{array}$ & 76.2 & 70 & 100 & 60 & 100 & 100 & 100 & 100 & 33.3 & 80 \\
\hline $\mathrm{DM}(\%)$ & 57.1 & 60 & 83.3 & 20 & 25 & 20 & 0 & 42.9 & 33.3 & 0 \\
\hline HT (\%) & 90.5 & 100 & 100 & 60 & 81.3 & 80 & 50 & 100 & 50 & 0 \\
\hline $\mathrm{DL}(\%)$ & 71.4 & 90 & 50 & 60 & 50 & 80 & 50 & 28.6 & 33.3 & 0 \\
\hline CAD (\%) & 28.6 & 20 & 33.3 & 40 & 50 & 60 & 50 & 42.9 & 33.3 & 0 \\
\hline Antiplatelet (\%) & 95.2 & 90 & 100 & 100 & 56.3 & 80 & 50 & 42.9 & 33.3 & 0 \\
\hline Statin (\%) & 61.9 & 80 & 50 & 40 & 50 & 80 & 50 & 28.6 & 33.3 & 0 \\
\hline ACE/AT II blockers (\%) & 71.4 & 70 & 83.3 & 60 & 37.5 & 60 & 25 & 28.6 & 50 & 0 \\
\hline$\beta$-Blockers (\%) & 38.1 & 50 & 33.3 & 20 & 43.8 & 60 & 25 & 42.9 & 33.3 & 0 \\
\hline Renal insufficiency (\%) & 0 & 0 & 0 & 0 & 6.25 & 0 & 0 & 14.3 & 0 & 0 \\
\hline Hepatic failure (\%) & 0 & 0 & 0 & 0 & 0 & 0 & 0 & 0 & 0 & 0 \\
\hline Sepsis (\%) & 4.8 & 0 & 0 & 20 & 0 & 0 & 0 & 0 & 0 & 0 \\
\hline Hypovolemic shock (\%) & 0 & 0 & 0 & 0 & 0 & 0 & 0 & 0 & 0 & 0 \\
\hline Acute heart failure (\%) & 0 & 0 & 0 & 0 & 0 & 0 & 0 & 0 & 0 & 0 \\
\hline
\end{tabular}

DM=Diabetes Mellitus, HT=Hypertension, DL=Dyslipidemia, CAD=Coronary Artery Disease.

AA, ATH and TAB groups were matched for gender, age, medication, history as well as renal and liver function tests. HBD group, although matched with ATH and AA groups for gender and smoking status, represents a different population group that is approximately 25 years younger. 


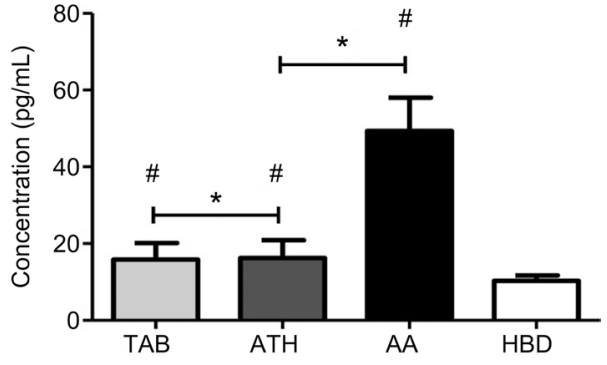

Figure 1. Relaxin 2 serum levels in all study groups. Group definitions: TAB (temporal artery biopsy); ATH (atherosclerosis); AA (arterial aneurysm); HBD (healthy blood donors). ${ }^{*} P<0.05$ between groups; ${ }^{\#} P<0.05$ between groups (TAB, ATH, AA) and HBD. Data are presented as the mean $\pm S D$.

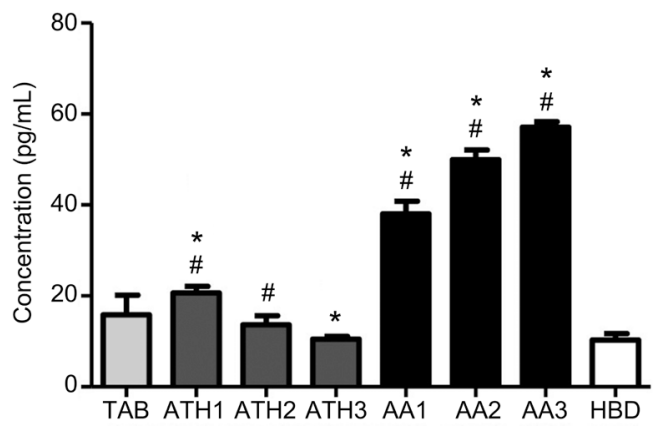

Figure 2. Relaxin 2 serum levels in subgroups. Serum relaxin 2 increased with the size or symptom status of the aneurysm, while it decreased with the clinical progression of atherosclerosis. Data are presented as the mean \pm SD. ${ }^{*} P<0.05$ between groups (ATHx, AAx) and TAB; ${ }^{\#} P<0.05$ between groups (ATHx, AAx) and HBD.

RL2 was comparable to HBD $(P=0.788)$. The TAB group's serum RL2 was comparable to the ATH2 group $(P>0.05)$.

\section{Discussion}

During the last two decades, several biomarkers have been investigated for a potential relationship with the development and progression of aneurysms in an effort to optimize timely diagnosis ${ }^{[26,27]}$. Matrix metalloproteinases, MMP-9 in particular, are among the most extensively studied biomarkers ${ }^{[28]}$. The relationship between MMPs and RL2 $2^{[3,4,10]}$ was the rationale behind the present study in patients with an arterial aneurysm. It was evident that patients with a known AA requiring surgical treatment had substantially higher serum RL2 than ATH patients or control subjects. In addition, serum RL2 increased proportionally to the increase in aneurysm size, exhibiting the highest levels in either a symptomatic aneurysm of any diameter or an asymptomatic aneurysm of a diameter $>350 \%$ of that of the normal artery. To the best of our knowledge, this is the first study to correlate the serum RL2 level with the size and existence of an aneurysm.

There have been a few reports suggesting the involvement of RL2 in the atherosclerotic process ${ }^{[13,18,20-23]}$, showing a potential beneficial effect on vascular inflammation ${ }^{[21]}$, vascular oxidative stress, vasorelaxation, and atherosclerotic plaque development ${ }^{[23]}$. Based on the current knowledge, we hypothesized that RL2 would be decreased in atherosclerotic patients to a level significantly lower than that of healthy individuals. This hypothesis was not verified; on the contrary, we found that serum RL2 was higher at early stages of atherosclerosis and gradually decreased with the disease progress, eventually exhibiting comparable results between patients with permanent ischemic manifestations from target organs (ATH3 group) and HBD. Interestingly, a previous study showed beneficial results from oral administration of porcine relaxin in patients with peripheral artery disease $\mathrm{e}^{[14]}$. Based on that, our findings could imply that RL2 compensates for the decreased blood supply in the early stages of atherosclerosis, a mechanism that probably becomes ameliorated in late clinical stages of the disease. However, further mechanistic insights on the underlying mechanisms of this effect were not investigated in this study.

The TAB group was initially included in the design of our study as a control group for both ATH and AA patients. Nevertheless, comparisons with the ATH group should be performed with caution, as two out of six patients in the $\mathrm{TAB}$ group had a previous history of coronary artery disease (CAD). These patients were free of CAD symptoms and were included in the study since CAD was not considered an exclusion criterion. We are not aware of any study on the role of RL2 in human subjects with CAD. In view of our results on the relationship of serum RL2 in ATH patients with extracranial carotid artery disease and peripheral arterial disease, we believe that relaxin might be related to CAD as well. However, due to the limited number of patients in this group, no safe conclusions can be drawn. Mores studies with CAD patients further subdividing these patients according to the clinical severity of their disease would be appropriate to investigate a potential role of relaxin in CAD.

Our study is not without limitations. First, the small study sample, the fact that the HBD group was not matched with other groups for comorbidities and the heterogeneous pathologies among patient groups, especially in the ATH group, warrant caution when interpreting the results and drawing further conclusions. Second, our results are purely descriptive, with no mechanistic insights on the underlying mechanisms of RL2 in the aforementioned vascular diseases. In order to clarify its potential applicability as a novel therapeutic target, the current literature provides limited data on possible RL2 mechanisms of action in arterial aneurysms. In a recent study, relaxin failed to augment angiotensin II (Ang II)-induced abdominal aortic aneurysms in mice ${ }^{[29]}$. The possibilities for the role of RL2 in atherosclerosis include stimulation of vasculogenesis through the upregulation of vascular endothelial growth factor (VEGF) transcript ${ }^{[30-34]}$, promotion of vasodilation through NO production ${ }^{[35-41]}$, reduction of systemic arterial resistance and increase of global artery compliance ${ }^{[42-47]}$, prevention of vascular dysfunction and amelioration of increased responsiveness to Ang $\mathrm{II}^{[48]}$.

In conclusion, serum RL2 is higher in patients with an arterial aneurysm, showing a positive relationship with the size 
of the aneurysmatic dilatation. The RL2 level is inversely correlated with the severity of the atherosclerotic disease. Studies with a larger cohort incorporating a consistent study population are warranted to verify our results and shed light on the mechanistic background of these processes.

\section{Author contribution}

Konstantinos PAPOUTSIS and Sotirios GEORGOPOULOS designed the study; Konstantinos PAPOUTSIS, Ioannis KARAVOKYROS, and Sotirios GEORGOPOULOS acquired the data; Alkistis KAPELOUZOU analyzed and interpreted data; Konstantinos PAPOUTSIS, Alkistis KAPELOUZOU, Diamantis I TSILIMIGRAS drafed the manuscript; Konstantinos PAPOUTSIS, Alkistis KAPELOUZOU, Diamantis I TSILIMIGRAS, Nikolaos PATELIS, Georgios KOUVELOS, Dimitrios SCHIZAS, Ioannis KARAVOKYROS, Sotirios GEORGOPOULOS made critical revision: Alkistis KAPELOUZOU and Sotirios GEORGOPOULOS supervised the study.

\section{References}

1 Bani D, Bigazzi M. Relaxin as a cardiovascular drug: a promise kept. Curr Drug Saf 2011; 6: 324-8.

2 James R, Niall H, Kwok S, Bryand-Greenwood G. Primary structure of porcinerelaxin: homology with insulin and related growth factors. Nature 1977; 267: 544-6.

3 Bathgate RA, Halls ML, van der Westhuizen ET, Callander GE, Kocan M, Summers RJ. Relaxin family peptides and their receptors. Physiol Rev 2013; 93: 405-80.

4 Feijóo-Bandín S, Aragón-Herrera A, Rodríguez-Penas D, Portolés M, Roselló-Lletí E, Rivera M, et al. Relaxin-2 in cardiometabolic diseases: mechanisms of action and future perspectives. Front Physiol 2017; 8: 599.

5 Wilkinson TN, Speed TP, Tregear GW, Bathgate RA. Evolution of the relaxin-like peptide family: from neuropeptide to reproduction. Ann $\mathrm{N}$ Y Acad Sci 2005; 1041: 530-3.

6 Siddle K. Signalling by insulin and IGF receptors: supporting acts and new players. J Mol Endocrinol 2011; 47: R1-10.

7 Paolocci N, Biondi R, Bettini M, Lee Cl, Berlowitz CO, Rossi R, et al. Oxygen radical-mediated reduction in basal and agonist-evoked NO release in isolated rat heart. J Mol Cell Cardiol 2001; 33: 671-9.

8 Novak J, Parry $\sqcup$, Matthews JE, Kerchner $\sqcup$, Indovina K, Hanley-Yanez $\mathrm{K}$, et al. Evidence for local relaxin ligand-receptor expression and function in arteries. FASEB J 2006; 20: 2352-62

9 Jelinic M, Leo CH, Post Uiterweer ED, Sandow SL, Gooi JH, Wlodek $\mathrm{ME}$, et al. Localization of relaxin receptors in arteries and veins, and region-specific increases in compliance and bradykinin-mediated relaxation after in vivo serelaxin treatment. FASEB J 2014; 28 : 27587.

10 Jeyabalan A, Novak J, Doty KD, Matthews J, Fisher MC, Kerchner LJ, et al. Vascular matrix metalloproteinase-9 mediates the inhibition of myogenic reactivity in small arteries isolated from rats after shortterm administration of relaxin. Endocrinology 2007; 148: 189-97.

11 Kapila S, Wang W, Uston K. Matrix metalloproteinase induction by relaxin causes cartilage matrix degradation in target synovial joints. Ann N Y Acad Sci 2009; 1160: 322-8.

12 Maruo N, Nakabayashi K, Wakahashi S, Yata A, Maruo T. Effects of recombinant $\mathrm{H} 2$ relaxin on the expression of matrix metalloproteinases and tissue inhibitor metalloproteinase in cultured early placental extravillous trophoblasts. Endocrine 2007; 32: 30310.

13 Goodall S, Crowther M, Hemingway DM, Bell PR, Thompson MM. Ubiquitous elevation of matrix metalloproteinase-2 expression in the vasculature of patients with abdominal aneurysms. Circulation 2001; 104: 304-9.

14 Goodall S, Porter KE, Bell PR, Thompson MM. Enhanced invasive properties exhibited by smooth muscle cells are associated with elevated production of MMP-2 in patients with aortic aneurysms. Eur J Vasc Endovasc Surg 2002; 24: 72-80.

15 Longo GM, Xiong W, Greiner TC, Zhao Y, Fiotti N, Baxter BT. Matrix metalloproteinases 2 and 9 work in concert to produce aortic aneurysms. J Clin Invest 2002; 110: 625-32.

16 Pyo R, Lee JK, Shipley JM, Curci JA, Mao D, Ziporin SJ, et al. Targeted gene disruption of matrix metalloproteinase-9 (gelatinase B) suppresses development of experimental abdominal aortic aneurysms. J Clin Invest 2000; 105: 1641-9.

17 Shah PK. Inflammation, metalloproteinases, and increased proteolysis: an emerging pathophysiological paradigm in aortic aneurysm. Circulation 1997; 96: 2115-7.

18 Balta S, Demirkol S, Celik T, Unlu M, Kucuk U, Arslan Z. Inflammatory markers should be assessed together with cardiovascular risk factors by clinicians in masked hypertension. J Clin Hypertens (Greenwich) 2013; 15: 443-4.

19 Brecht A, Bartsch C, Baumann G, Stangl K, Dschietzig T. Relaxin inhibits early steps in vascular inflammation. Regul Pept 2011; 166: 76-82.

20 Papadopoulos DP, Mourouzis I, Faselis C, Perrea D, Makris T, Tsioufis C, et al. Masked hypertension and atherogenesis: the impact of apelin and relaxin plasma levels. J Clin Hypertens (Greenwich) 2013; 15: 333-6.

21 Sonaglia F, Milia P, Caserio M, Bigazzi B, Bigazzi B, Ricotta S, et al. Efficacy and safety of oral porcine relaxin ( $p R L X)$ in adjunct to physical exercise in the treatment of peripheral arterial disease (PAD). Ital J Anat Embryol 2013; 118: 84-91.

22 Tiyerili V, Beiert T, Schatten H, Camara B, Jehle J, Schrickel JW, et al. Anti-atherosclerotic effects of serelaxin in apolipoprotein E-deficient mice. Atherosclerosis 2016; 251: 430-7.

23 North American Symptomatic Carotid Endarterectomy Trial. Methods, patient characteristics, and progress. Stroke 1991; 22: 711-20.

24 Rutherford RB, Baker JD, Ernst C, Johnston KW, Porter JM, Ahn S, et al. Recommended standards for reports dealing with lower extremity ischemia: revised version. J Vasc Surg 1997; 26: 517-38.

25 Johnston KW, Rutherford RB, Tilson MD, Shah DM, Hollier L, Stanley JC. Suggested standards for reporting on arterial aneurysms. Subcommittee on Reporting Standards for Arterial Aneurysms, Ad Hoc Committee on Reporting Standards, Society for Vascular Surgery and North American Chapter, International Society for Cardiovascular Surgery. J Vasc Surg 1991; 13: 452-8.

26 Moris D, Mantonakis E, Avgerinos E, Makris M, Bakoyiannis C, Pikoulis $\mathrm{E}$, et al. Novel biomarkers of abdominal aortic aneurysm disease: identifying gaps and dispelling misperceptions. Biomed Res Int 2014; 2014: 925840.

27 Urbonavicius S, Urbonaviciene G, Honore B, Henneberg EW, Vorum $\mathrm{H}$, Lindholt JS. Potential circulating biomarkers for abdominal aortic aneurysm expansion and rupture-a systematic review. Eur J Vasc Endovasc Surg 2008; 36: 273-80; discussion 81-2.

28 Takagi H, Manabe H, Kawai N, Goto SN, Umemoto T. Circulating matrix metalloproteinase- 9 concentrations and abdominal aortic aneurysm presence: a meta-analysis. Interact Cardiovasc Thorac Surg 2009; 9: 437-40. 
29 Howatt DA, Dajee M, Xie X, Moorleghen J, Rateri DL, Balakrishnan $\mathrm{A}$, et al. Relaxin and matrix metalloproteinase-9 in angiotensin IIinduced abdominal aortic aneurysms. Circ J 2017; 81: 888-90.

30 Shirota K, Tateishi K, Emoto M, Hachisuga T, Kuroki M, Kawarabayashi T. Relaxin-induced angiogenesis in ovary contributes to follicle development. Ann N Y Acad Sci 2005; 1041: 144-6.

31 Silvertown JD, Ng J, Sato T, Summerlee AJ, Medin JA. H2 relaxin overexpression increases in vivo prostate xenograft tumor growth and angiogenesis. Int J Cancer 2006; 118: 62-73.

32 Segal MS, Sautina L, Li S, Diao Y, Agoulnik Al, Kielczewski J, et al. Relaxin increases human endothelial progenitor cell $\mathrm{NO}$ and migration and vasculogenesis in mice. Blood 2012; 119: 629-36.

33 Bitto A, Irrera N, Minutoli L, Calò M, Lo Cascio P, Caccia P, et al. Relaxin improves multiple markers of wound healing and ameliorates the disturbed healing pattern of genetically diabetic mice. Clin Sci (Lond) 2013; 125: 575-85.

34 Unemori EN, Lewis M, Constant J, Arnold G, Grove BH, Normand J, et al. Relaxin induces vascular endothelial growth factor expression and angiogenesis selectively at wound sites. Wound Repair Regen 2000; 8: 361-70.

35 Bani G, Bani Sacchi T, Bigazzi M, Bianchi S. Effects of relaxin on the microvasculature of mouse mammary gland. Histol Histopathol 1988; 3: 337-43.

36 Vasilenko P, Mead JP, Weidmann JE. Uterine growth-promoting effects of relaxin: a morphometric and histological analysis. Biol Reprod 1986; 35: 987-95.

37 Bani G, Maurizi M, Bigazzi M, Bani Sacchi T. Effects of relaxin on the endometrial stroma. Studies in mice. Biol Reprod 1995; 53: 253-62.

38 Bigazzi M, Del Mese A, Petrucci F, Casali R, Novelli GP. The local administration of relaxin induces changes in the microcirculation of the rat mesocaecum. Acta Endocrinol (Copenh) 1986; 112: 296-9.

39 Danielson LA, Sherwood OD, Conrad KP. Relaxin is a potent renal vasodilator in conscious rats. J Clin Invest 1999; 103: 525-33.
40 Danielson LA, Kercher LJ, Conrad KP. Impact of gender and endothelin on renal vasodilation and hyperfiltration induced by relaxin in conscious rats. Am J Physiol Regul Integr Comp Physiol 2000; 279 : R1298-304.

41 Novak J, Danielson LA, Kerchner $\sqcup$, Sherwood OD, Ramirez RJ, Moalli $\mathrm{PA}$, et al. Relaxin is essential for renal vasodilation during pregnancy in conscious rats. J Clin Invest 2001; 107: 1469-75.

42 Conrad KP, Debrah DO, Novak J, Danielson LA, Shroff SG. Relaxin modifies systemic arterial resistance and compliance in conscious, nonpregnant rats. Endocrinology 2004; 145: 3289-96.

43 Debrah DO, Conrad KP, Danielson LA, Shroff SG. Effects of relaxin on systemic arterial hemodynamics and mechanical properties in conscious rats: sex dependency and dose response. J Appl Physiol 2005; 98: 1013-20.

44 Debrah DO, Conrad KP, Novak J, Danielson LA, Shroff SG. Recombinant human relaxin ( $r h R L X$ ) modifies systemic arterial properties in conscious rats irrespective of gender, but in a biphasic fashion. Ann N Y Acad Sci 2005; 1041: 155-62.

45 Debrah DO, Novak J, Matthews JE, Ramirez RJ, Shroff SG, Conrad KP. Relaxin is essential for systemic vasodilation and increased global arterial compliance during early pregnancy in conscious rats. Endocrinology 2006; 147: 5126-31

46 Conrad KP, Shroff SG. Effects of relaxin on arterial dilation, remodeling, and mechanical properties. Curr Hypertens Rep 2011; 13: 409-20.

47 Xu Q, Chakravorty A, Bathgate RA, Dart AM, Du XJ. Relaxin therapy reverses large artery remodeling and improves arterial compliance in senescent spontaneously hypertensive rats. Hypertension 2010; 55: 1260-6.

48 Marshall SA, Leo CH, Girling JE, Tare M, Beard S, Hannan NJ, et al. Relaxin treatment reduces angiotensin II-induced vasoconstriction in pregnancy and protects against endothelial dysfunction. Biol Reprod 2017; 96: 895-906. 